\title{
The Characteristics of Methylphenidate on Animal Behavior
}

\section{Nachum Dafny}

Department of Neurobiology and Anatomy, The University of Texas Medical School at Houston

\begin{abstract}
Although methylphenidate has been used for decades as a leading treatment for behavioral disorders such as attention deficit hyperactivity disorder (ADHD). Recently it has been used as a cognitive enhancer and for recreation. The mechanism underlying its actions is still unrevealed. Enhancement of mental function by prescription psychostimulants that promise to improve mental performance, attention, memory, planning, etc., is now wide spread. Moreover, in the last two decades stimulants such as MPD have been prescribed to very young children and adults for treating behavioral disorders such as ADHD. This study aims to provide a short review of methylphenidate's effect on the animal's behavior.
\end{abstract}

Keywords: Psychostimulant; Ritalin; Behavior; Sensitization; Circadian rhythm; Cognitive enhancer

\section{Introduction}

Pyschostimulant medication such as amphetamine was used to treat attention deficit hyperactive disorder (ADHD) patients from 1930's until it was found it elicits dependency. The psychostimulants methylphenidate (MPD) has since become the drug of choice to treat ADHD patients [1]. MPD is a CNS stimulant that closely relates to the structure of dextroamphetamine [2], a derivative of amphetamine. The neuropharmacological profile of MPD is also similar to that of cocaine [2]. The drug was first synthesized in 1944 and used initially as an analeptic for several types of barbiturate-induced coma. It was later used as a drug to improve memory in elderly patients. Since then, its usage has been extended to improve the alertness in children and adults with emotional, behavioral, and learning difficulties [1]. MPD is highly effective in treating ADHD [3] and useful in providing relief from intractable pain in narcolepsy and chronic fatigue.

Several articles reported that people deserve the right to boost their brains with psychoactive pills, like those prescribed for Attention Deficit Hyperactivity Disorder (ADHD), narcolepsy and memory-impaired older folks. Students of all ages are already taking MPD (Ritalin), to help them study and perform in exam better. Some students contend that: We should welcome new methods of improving our brain function and doing it with pills is no more morally objectionable than healthy eating, taking vitamins or getting a good night's sleep with the help of a remedy. But what do we know about this psychoactive drug? Psychoactive drug use dates back to prehistoric times: there is archaeological evidence for their use as much as several thousand years ago. A psychoactive drug acts in the central nervous system (CNS) to modulate consciousness, perception, mood and behavior. These drugs were used therapeutically as medication or for ritual and spiritual purposes as well recreationally to alter one's mood and to get 'high'. Because psychoactive drugs elicit changes in consciousness and mood, the user feels alert, joyful, pleasant, and becomes euphoric. Many psychoactive drugs are used and abused despite the risks of its consequences, such as dependence. Methylphenidate belongs to this family of psychoactive drugs.

Clinically MPD is given orally, it is absorbed from the intestinal tract and has a half-life of about 1 hour with equally short duration and efficacy, while its peak level following injection (systemic) is faster and is reached peak level at $8-20$ minutes [4], which is similar to systematic cocaine and amphetamine administration [5]. MPD given systematically binds with similar affinity at the dopamine transporter
(DAT) and has potency $(\mathrm{Ki}=200)$ similar to cocaine $(\mathrm{Ki}=224)$ [4]. The relationship between drug doses (milligrams of hydrochloride salt/kilograms of body weight) and percentage occupancy of DAT is identical for cocaine and MPD in rodents and humans [2]. The dose and route of MPD administration are important because the features of the behavioral and neurochemical repsonses to the drug are dependent on the speed of the drug to reach peak level, i.e., the rise time of drug concentration. Peak levels of MPD following intravenous (i.v.), intraperitoneal (i.p.), and oral administration was 8-20 min, 15-28, and 60-90 min, respectively [5]. Similar peak levels of i.v., i.p. and oral administrations were obtained following amphetamine and cocaine [5]. The ability to reach peak levels in short spans of time (i.e. 8-30min) is one of the main factors in eliciting adverse effects such as sensitization. MPD is absorbed and metabolized via deethterification to ritalinic acid and released into the urine within 48 hours. Brain concentration of MPD exceeds that of plasma since the psychostimulants are concentrated in catecholaminergic systems with free passage across the blood brain barrier. MPD has a similar uptake in the brain as cocaine but differs from cocaine by having a much lower rate of clearance from the brain. Intravenous (i.v.) or intranasal administration of MPD has a higher mortality rate than that of cocaine or amphetamine. The outcome of MPD treatment resulted primarily by inhibiting the re-uptake of dopamine (DA), norepinephrine (NE), and serotonin $(5 \mathrm{HT})$ from the synaptic cleft to the presynaptic terminals. Like cocaine, MPD is an indirect catecholamine (CA) agonist since it does not stimulate the catecholaminergic receptors directly but rather facilitates the action of the CA [2] The therapeutic effect of MPD in the treatment of $\mathrm{ADHD}$ has been attributed to its ability to increase the efflux of these neurotransmitters. This causes increases in extracellular DA, NA, and 5HT levels [4], which has an effect that has been linked to its reinforcing properties. MPD has moderate effects on the peripheral

*Corresponding author: Nachum Dafny, Professor, Department of Neurobiology and Anatomy, Director, Medical Neuroscience Course, The University of Texas Health Science Center at Houston, 6431 Fannin Street, Suite 7.208Houston, Texas 77030, Tel: (713)500-5616; Fax: (713)500-0621; E-mail: Nachum.Dafny@uth.tmc.edu

Received January 22, 2015; Accepted August 18, 2015; Published August 21 2015

Citation: Dafny N (2015) The Characteristics of Methylphenidate on Animal Behavior. Pharm Anal Acta 6: 404. doi:10.4172/21532435.1000404

Copyright: $(2015$ Dafny N, et al. This is an open-access article distributed under the terms of the Creative Commons Attribution License, which permits unrestricted use, distribution, and reproduction in any medium, provided the original author and source are credited. 
circulatory system. In rats, MPD administration in low doses (1-3 mg/ $\mathrm{kg}$ ) stimulates locomotor activity and in high doses $(10 \mathrm{mg} / \mathrm{kg}$ and higher) produce stereotypical behavior and increased locomotion [6].

Methylphenidate has been abused for both 'cognitive enhancement' and recreational purposes to get 'high' in addition to improve attention and control misbehavior, increase wakefulness and task focusing. Their euphoric effects usually occur when they are crushed and snorted or injected.

\section{Animal assay to study the behavioral effect of Psychostimulant}

One of the most used assays to study animal behavior is the open field assay (Figure 1). Open field assays were introduced more than 80 years ago, it resembles a natural behavioral pattern and is one of the most widely used methods in animal behavioral research. Its popularity stems from the simplicity of the apparatus and of clearly defined behaviors. Certain measurable motor behaviors are sensitive to a wide range of drugs and experimental manipulations and are sufficiently reliable under standardized conditions to give repeatable measures on an enormous range of independent variable. Simplicity, reproducibility (Figure 2), ease of quantification, and wide applicability are the prime determinant of its popularity. Open-field locomotor behaviors represent the interaction of the subject with the experimental situation. Most investigators study the effects of psychostimulants in open-field testing whether repetitive (chronic) drug exposure elicits behavioral tolerance or sensitization [6,7]. Since drug liability is defined mainly on the basis of the subject behavioral expression to chronic drug exposure such as behavioral withdrawal, sensitization or tolerance. Behavioral expressions after psychostimulants administration develop gradually and progressively during the course of repeated exposure of psychostimulants, and this expression can persist for long periods of time after its discontinuation [8]. Thus, withdrawal, sensitization and tolerance are considered a form of drug induced neuronal plasticity and are used as an experimental model and as a marker for drugs effects, and for its liability of abuse. Behavioral tolerance is defined by the following criterion: repetitive exposure with the same dose of MPD will become less effective to elicit its initial effects. Behavioral sensitization is defined as reverse tolerance i.e., repetitive exposure with the same MPD dose will elicit a significant increase in its behavioral effects compared to that elicited by the initial (acute) dose (Figure 3).

Behavioral sensitization and/or tolerance are considered a long lasting neuroplasticity elicited following repetitive psychostimulant exposure. It has been suggested that behavioral tolerance and sensitization represent an enduring alteration of drug response and have been used as an experimental model of drug craving [9]. It was reported that the same repetitive dose of MPD in some animals elicited behavioral sensitization and in others behavioral tolerance. Moreover, the neuronal recording from central nervous system (CNS) structures belonging to the brain motive/reward circuit recorded from animals expressing behavioral sensitization to chronic MPD exposure responded to MPD in significantly different ways compared to those animals expressing behavioral tolerance to chronic MPD exposure [1012]. Since chronic MPD use can elicit either behavioral sensitization or tolerance, psychostimulant therapy given to adolescents and young adults may increase the risk for Substance Use Disorder [10], while other reports suggest that psychostimulant treatment in adolescents with ADHD protects them from later Substance Use Disorder. These contradictory reports call for basic in-depth studies to resolve this critical issue. Animal models using behavior and neuronal recordings following acute and chronic methylphenidate treatment can be helpful in this respect.
Repetitive (chronic) treatment with psychostimulants to children, such as MPD and amphetamine, can modulate the neurodevelopmental processes critically. It has been reported that drugs such as MPD modulate the circadian rhythm as a result of molecular alteration of clock genes [14]. The alteration of the circadian activity behavior alters the body's homeostasis. There is some concern about children with ADHD who are going through these neurodevelopmental processes while being treated with MPD for extended periods of time.

\section{What is the best animal model to study the effect of methylphenidate}

Key questions are which animal and which strain should be used to study the physiological properties of MPD? Obviously, the most appropriate animal model is the one that best mimics ADHD in

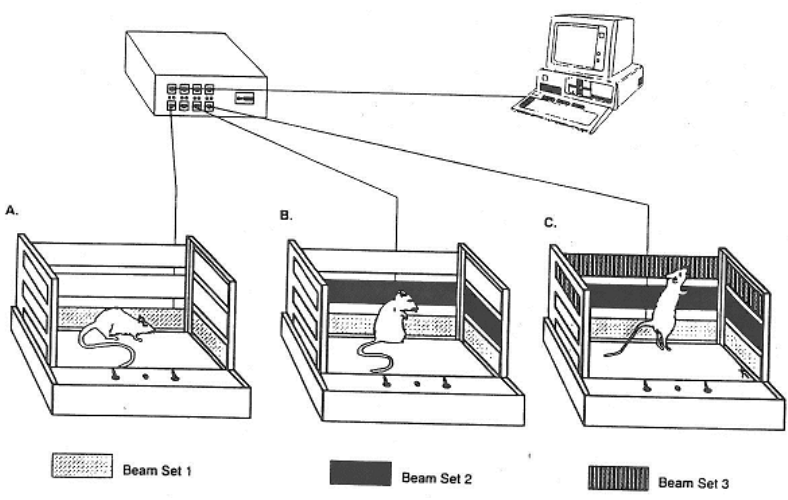

Figure 1: The figure shows the cage with three different levels of pane (marked in black. The cage is $40 \mathrm{~cm}$ in length, $40 \mathrm{~cm}$ in width and $30 \mathrm{~cm}$ high. Each panel has 16 infra-red beams and in the opposite side are their sensors. The panels are located 5,10 , and $15 \mathrm{~cm}$ above the floor of the cage. The lower panels record the total movement horizontal activity and the total distance traveling of the animals. The second level of panels with its sensors records the number of stereotypic movement and the third level records the vertical activity before and after drug exposure.

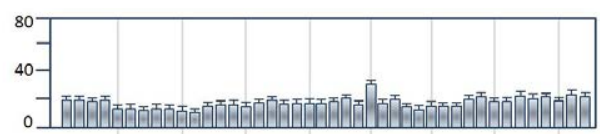

Counts $/ 12 \mathrm{~h}$ light

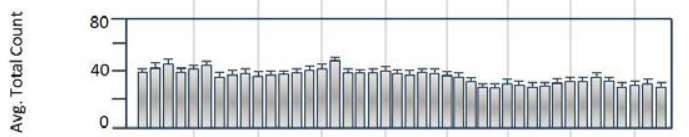

Counts $/ 12 \mathrm{~h}$ night

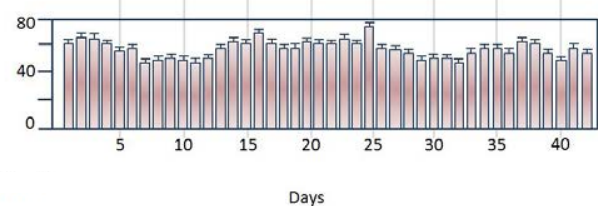

Counts $/ 24 \mathrm{~h}$

Figure 2: The figure demonstrates the stability of the baseline activity recorded from adult $\mathrm{SD}$ male rats $(\mathrm{N}=8)$ over 42 sequential days. The upper (A), middle (B) and lower (C) histograms sum the total activity during the light period (12 hrs), during the light period and during 24 hours respectively. In D is the weekly total activity for six consecutive weeks. The figures demonstrate the stability of the measurement with minor non-significant fluctuations as expected. 

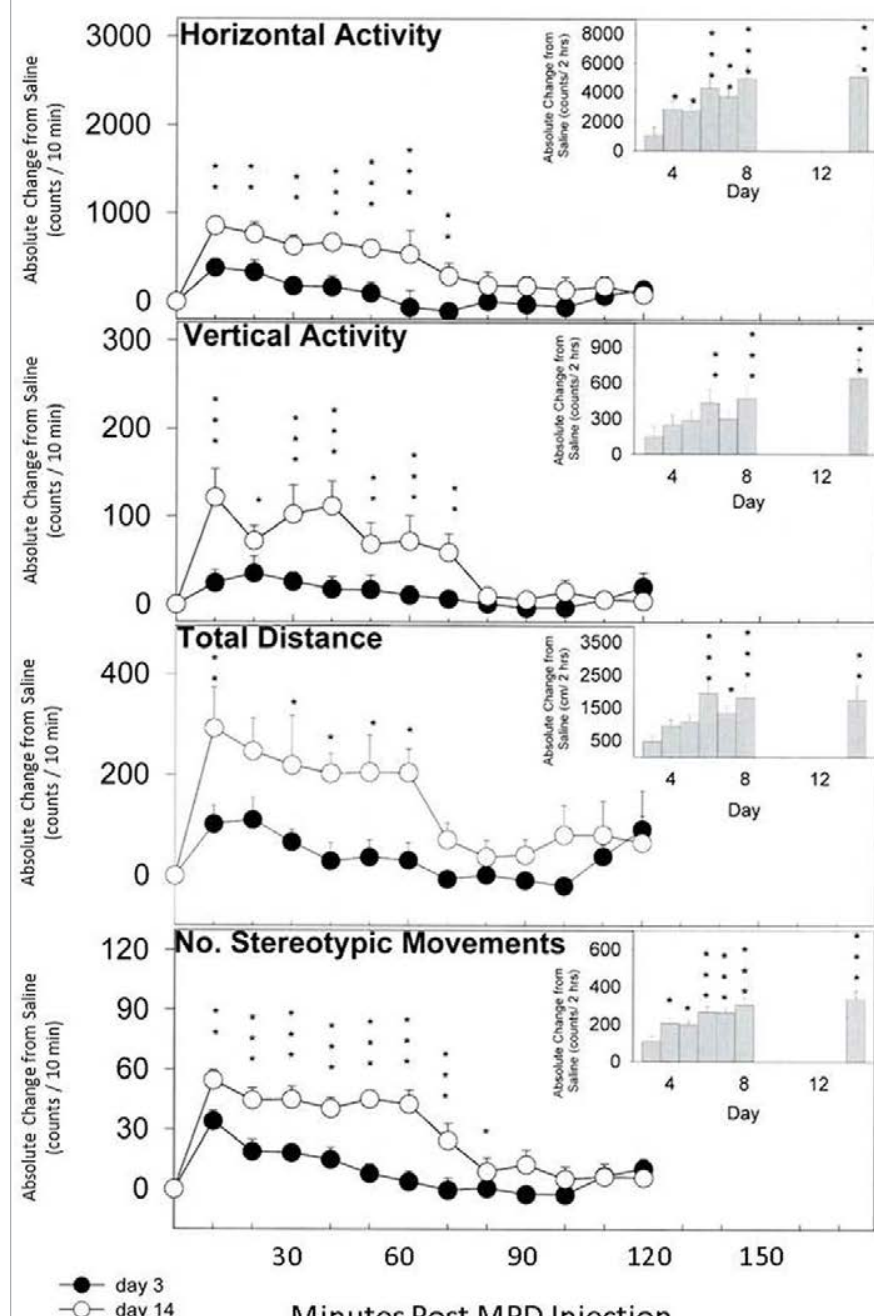

day 14

Minutes Post MPD Injection

Figure 2: The figure summarizes $(\mathrm{N}=8)$ the effect of acute and chronic effect of $2.5 \mathrm{mg} / \mathrm{kg}$ methylphenidate (MPD) on Horizontal activity, Total Distance Traveling, and number of stereotypic movement. The black circle sums the activity/ $10 \mathrm{~min}$ after the initial (acute) MPD injections, the white circle sums the activity at experimental day 11 after six daily MPD exposure and 3 washout days. The histogram in the upper right corner depicts the total change from two baseline days activity (set arbitrarily at 0 ) and six daily MPD exposure, 5 days of washout and MPD rechallenge at day 14 . The numbers indicate the experimental days. * indicates significant $(p<0.05)$ from the initial effect of MPD. The figure shows that repetitive (chronic) MPD elicited behavioral sensitization.

humans and is able to predict aspects of ADHD behavior. There are differences between different animal strains in their susceptibility to psychostimulants and their chronic effects, such as tolerance or sensitization (Figure 4). Since no biological marker for ADHD has yet been identified, diagnosis of ADHD is presently based on behavioral symptoms alone. Many suggested animal models of ADHD exist. These include rats that: are outbred from a general colony [15]; reared in social isolation; have been exposed to environmental pollutants; have undergone neonatal anoxia, hippocampal x-irradiation in infancy, or selective neurotoxic lesion of DA, NE, etc. neurons; and genetically mutant mice. There are also inbred strains, including Naples High/ Low excitability and Spontaneously Hypertensive Rat (SHR) strain, the latter of which was bred from progenitor Wistar Kyoto (WKY) rats [16]. The SHR is hyperactive with a variety of behavioral characteristics that are comparable to the behavior of children with ADHD, including motor and cognitive impulsiveness, impaired attention, and hyperactivity [16]. Therefore, the SHR strain is used most often in ADHD/methylphenidate studies.

\section{Age-dependent effects of methylphenidate}

The ontogeny of the brain/behavior relationship during the period between preadolescence, adolescence and attained sexual maturity needs more attention. Based on many reports, the following age classification can be derived: from postnatal day 21 (P-21) to P-30, P-31 to $\mathrm{P}-39, \mathrm{P}-40$ to $\mathrm{P}-50, \mathrm{P}-60$ to $\mathrm{P}-75$ and $\mathrm{P}-76$ and above the rats are considered as juveniles, periadolescents, adolescents, young adult and adult rats, respectively (Table 1 ).

The response to psychostimulants varies with age in humans and other animals [9]. During normal development, overproduction of synaptic connections and receptors occurs, which is followed by their pruning or competitive elimination. The marked overproduction and elimination of synapses and receptors during adolescence may serve as a permissive factor for a number of behavioral/psychiatric disorders, including ADHD [17]. Between 5 and 15 years of age in humans, DA synaptic density in the frontal cortex decreases by approximately $40 \%$. This phenomenon can be modified by chronic MPD in the young, which could cause some undesired behavioral disorders. The time-course and nature of this phenomenon parallels the ADHD time-course as a result of alteration or over-production and regressive synaptic elimination described above.

Adolescent and adult rats are affected differently by catecholaminergic agonists. Whereas adolescent rats exhibit an attenuated behavioral response, compared to adult rats, while adult rats exhibit a greater behavioral response to psychostimulants compared to adolescent rats [18]. Rats exposed to MPD during the period equivalent to human adolescence display behavioral changes that endure into adulthood. This suggests that MPD has a neurobiological effect in adolescents that modulates the 'normal' development to adulthood [15,9].

Studies of amphetamine and cocaine sensitization in developing animals have yielded conflicting results, depending upon the age at the time of testing, the intervals between the repetitive drug treatment, and the challenge dose [18]. Adolescent rats of both sexes show sensitization to locomotor activating effects of cocaine, whereas different locomotor sensitization profiles were found in adult rats [18]. However, others have reported that younger animals treated chronically with psychostimulants rarely exhibit sensitization and that, even when sensitization occurs, it persists for a shorter period of time. When the effects of chronic cocaine were compared in adolescent and adult rats the former showed alterations in psychopharmacological sensitivity. These apparently did not rely on age-specific decreases in brain drug availability but rather appeared to be related to alterations in CNS sensitivity [18].

\section{Gender differences in the effects of methylphenidate}

Biomedical investigations has been conducted almost exclusively with male subjects. The reason for excluding females as subjects in research is that they have greater biological complexity than males due to their reproductive cyclicity $[19,20]$. It has only recently become evident that the gonadal steroid hormones have multiple functions [20]. The institute of Medicine concluded that "...the understanding of sex differences in health and illnesses merits serious scientific inquiry in all aspects of biomedical and health-related research". Furthermore, sex-related differences are often controversial and not documented. 


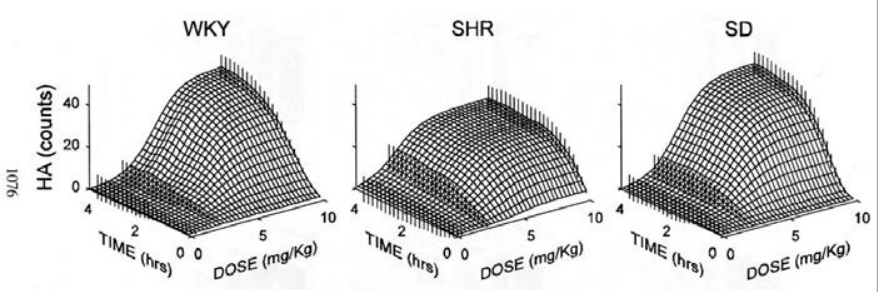

Figure 4: The figure shows the temporal area under the curve for horizontal activity following MPD doses $(0.6,2.5$, and $10.0 \mathrm{mg} / \mathrm{kg}$ i.p.). The temporal responses of WKY and SD rats are similar patterns following the three MPD exposures, while the SHR strain rats expresses different response patterns.

\begin{tabular}{|l|l|l|l|l|}
\hline Juvenile & Periadolescent & Adolescent & Young adult & Adult \\
\hline P-21 to P-30 & P-31 to P-39 & P-40 to P-50 & P-60 to P-75 & P->75 \\
\hline
\end{tabular}

Table 1: Stages of development of the postnatal rat.

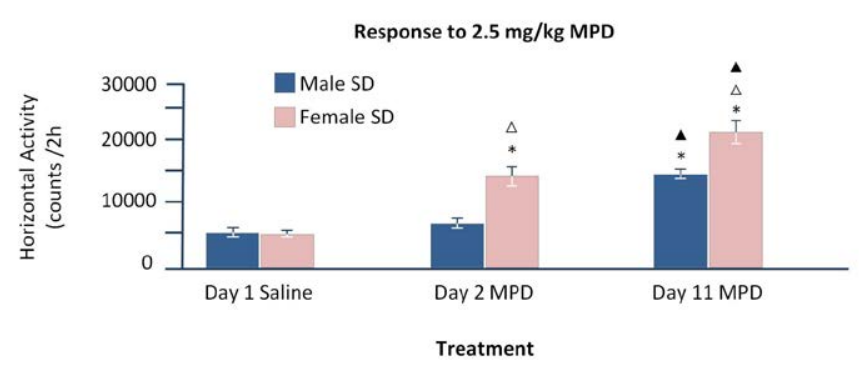

Figure 5: The figure summarizes the horizontal activity of male and female $\mathrm{SD}$ rats (each group $\mathrm{N}=8$ ) and demonstates that female rats exhibited higher intensity of behavioral sensitization than male rat groups. ${ }^{*} p<0.05$ compared to day 1 saline; $\Delta=p<0.05$ comparing male to female; $\boldsymbol{\Delta}=$ comparing day 11 to day 2.

Differences in the response to cocaine, amphetamine and MPD in response to repetitive exposure (Figure 5) may be due to sex differences in drug pharmacokinetics, particularly drug metabolism. The neural systems mediating the behavioral response to psychomotor stimulants are sexually dimorphic and the gonadal hormones are postulated to have important implications for gender differences in the acute and chronic responses to psychostimulants and in the susceptibility of addiction to these drugs. There are also remarkable gender differences in the behavioral expression of ADHD patients. For example, ADHD is more often diagnosed in males than in females and is 2-to-9 fold more prevalent in males. Females with ADHD may be more severely affected than males [21] as female ADHD subjects tend to have a higher genetic loading for the disorder. Anderson and Teicher (2001) hypothesized that there is an extensive overproductive of DA receptors in the male striatum and NAc during pre-pubertal development, which may help to explain why males are often more afflicted with ADHD because dopaminergic activity increases in these regions can produce hyperactivity and stereotypical behavior. Sex differences in ADHD may also be attributed to sex differences in DA receptor density. Striatal D2 receptor density in males increases $144 \% \pm 26 \%$ between 25 to 40 days, while females D2 receptor density increases only $31 \% \pm 7 \%$. The rise in males' striatal DA receptors parallels early development of ADHD motor systems [17].

In general, females were more sensitive than males to cocaine. The development of behavioral sensitization to cocaine was a function of sex-specific alterations in sensitivity to psychostimulants. In addition, accumulating evidence indicates that the antecedents, consequences, and mechanisms of drug abuse and addiction are different in female and males, and suggests that sex based research is an important variable to be considered in studying mechanisms and treatment and will provide more effective prevention and treatment strategies. It was reported that adult female rats are more seriously addicted to psychostimulants and express a more rapid and robust behavioral response to acute cocaine and amphetamine. Also, they usually display a greater and more rapid behavioral sensitivity to chronic exposure to these drugs compared to their male counterparts $[22,23]$. This sexual dimorphism was only observed in adult rats, suggesting that gonadal hormones secreted in adulthood might modulate the responsiveness to psychostimulants.

\section{Acknowledgement}

Acknowledgements I would like to thank Mallinckrodt Inc. for their gift of methylphenidate, and Dr. P.B. Yang, and C.M. Claussen for manuscript preparation. Supported in part by NIH DA R01 027222.

\section{References}

1. Godfrey J (2009) Safety of therapeutic methylphenidate in adults: a systematic review of the evidence. J Psychopharmacol 23: 194-205.

2. Gatley SJ, Volkow ND, Gifford AN, Fowler JS, Dewey, SL (1999) Dopaminetransporter occupancy after intravenous doses of cocaine and methylphenidate. J Am Acad Child Adolesc Psychiatry 37: 1242-1243.

3. Challman TD, Lipsky JJ (2000) Methylphenidate: its pharmacology and uses Mayo Clin 75: 711-721.

4. Kuczenski R, Segal DS (1997) Effects of methylphenidate on extracellular dopamine, serotonin, and norepinephrine: comparison with amphetamine. J Neurochem 68: 2032-2037.

5. Gerasimov MD, Franceschi, M, Volkow ND, Gifford A, Gatley SJ (2000) Comparison between intraperitoneal and oral methylphenidate administration: A microdialysis and locomotor activity study. J Pharmacol Exp Ther 296: 51-57.

6. Gaytan O, al-Rahim S, Swann A, Dafny N (1997) Sensitization to locomotor effects of methylphenidate in the rat. Life Sci 61: 101-107

7. Yang PB, Amini B, Swann AC, Dafny N (2003) Strain differences in the behavioral responses of male rats to chronically administered methylphenidate. Brain Res 971: 139-152.

8. Askenasy EP, Taber KH, Yang PB, Dafny N (2007) Methylphenidate (Ritalin): Behavioral Studies in the Rat. Intern. J Neurosci 117: 1-38.

9. Dafny N, Yang PB (2006) The role of age, genotype, sex, and route of acute and chronic administration of methylphenidate: A review of its locomotor effects. Brain Res Bull 68: 393-405.

10. Claussen CM, Chong SL, Dafny N (2014) Nucleus accumbens neuronal activity correlates to the animals behavioral response to acute and chronic methylphenidate. Physiol Behav 129: 85-94.

11. Jones Z, Dafny N (2014) Acute and chronic dose-response effect of methylphenidate on ventral tegmental area neurons correlated with animal behavior. J Neural Transm 121: 327-345.

12. Tang B, Dafny N (2013) Behavioral and dorsal raphe neuronal activity following acute and chronic methylphenidate in freely behaving rats. Brain Res Bull 98: 53-63.

13. Van Emmerik-van Oortmerssen K, van de Glind G, van den Brink W, Smit F Crunelle CL (2013) Prevalence of attention-deficit hyperactivity disorder in substance use order patients: a meta-analysis and meta-regression analysis. Drug Alcohol Depend 122: 11-29.

14. Algahim MF, Yang PB, Wilcox VT, Burau KD, Swann AC (2009) Prolonged methylphenidate treatment alters the behavioral diurnal activity pattern of adult male Sprague-Dawley rats. Pharmacol Biochem Behav 92: 93-99.

15. Barron E, Yang PB, Swann AC, Dafny N (2009) Adolescent and adult male spontaneous hyperactive rats (SHR) respond differently to acute and chronicmethylphenidate (Ritalin). Int J Neurosci 119: 40-58.

16. Sagvolden T, Lamm MC, Taljaard JJ (2000) Methylphenidate affects striata dopamine differently in an animal model for attention-deficit/hyperactivity disorder--the spontaneously hypertensive rat. Brain Res Bull 53: 187-192. 
17. Andersen SL, Teicher MH (2000) Sex differences in dopamine receptors and their relevance to ADHD. Neurosci Biobehav Rev 24: 137-141.

18. Laviola G, Wood RD, Kuhn C, Francis R, Spear CR (1995) Cocaine sensitization in periadolescent and adult rats. The J of Pharmacol and Exp Therap 275: 345-351.

19. Kelly SJ, Ostrowski NL, Wilson MA (1999) Gender differences in brain and behavior 64: 655-664.

20. McEwen BS, Alves SE, Bollch K, Weiland NG (1998) Clinically relevant basic science study of gender differences and sez hormone effects. Pyschopharm Bull 34: 251-259.
21. Biederman J, Faraone SV, Spencer T, Wilens T, Nick E (1994) Gender differences in a sample of adults with attention deficit hyperactivity disorder Psyciatry Res 53: 13-29.

22. Van Harran E, Meyer M (1991) Sex differences in locomotor activity after acute and chronic cocaine administration. Pharmacol Biochem Behav 39: 923-927.

23. Volkow ND, Wang GJ, Fowler JS, Fischma, M, Foltin R (1999) Methylphenidate and cocaine have a similar in vivo potency to block dopamine transporters in the human brain. Life Sci 65: 7-12. 